\title{
Consideraciones sobre el Derecho Indiano
}

\author{
Por MANUEL BELALINDE GUINASSI
}

Catedrático de las Facultades de Derecho y Letras.

Dedico este trabajo a mis alumnos de la Facultad de Derecho de la Universidad Católica del Perú.

SUMARIO:

$$
\begin{aligned}
& \text { I. - Contenido y Finalidad. } \\
& \text { II. - Periodos. } \\
& \text { III. - El Caso del Perú. }
\end{aligned}
$$

\section{I}

No se trata de un trabajo de investigación la exposición que se hace, sino simplemente el resultado de uno de los aspectos de las clases dictadas en la Universidad Católica del Perú, por tánto, su valor, es el de un artículo de extensión universitaria.

Conviene de manera especial situar en el tiempo, el conjunto de preceptos juridicos que forman el llamado Derecho Indiano, para así, poder apreciar con toda exactitud, la serie de factores, tanto espirituales como materiales, que en cada momento histórico determinaron su desarrollo, caracteristicas y consecuencias importantes.

El Descubrimiento de América, es el punto inicial para el Derecho Indiano, como también lo es, para la aplicación del Derecho Castellano; luego su nacimiento y posterior evolución se vinculan estrechamente a la conquista y colonización de las nuevas tierras, y podiamos decir que su existencia se prolonga, todo el tiempo de la dominación española, hasta la Independencia de las distintas regiones que formaron los dominios españoles en América, - sin dejar de reconocer que muchas de sus normas quedaron vigentes, por algún tiempo, después de la Emancipación. 
Situado en el tiempo, el cuerpo de normas juridicas que se dictaron por España para las Indias Occidentales, queda por establecer, sino la definición, por lo menos el concepto de lo que es el Derecho Indiano, en el panorama del desarrollo histórico del Derecho Americano y en la evolución juridica de los derechos nacionales de Hispano América.

El conocido investigador de la Historia del Derecho Español don José María Ots Capdequi, nos dá una visión simple, pero exacta, de lo que representa el Derecho Indiano, cuando dice:

“En cuanto al Derecho propiamente indiano. está integrado por "aquellas normas juridicas - Reales Cédulas. Provisiones. Instruc-

"ciones, Ordenanzas, etc. - que fueron dictadas por los Monarcas

" españoles o por sus autoridades delegadas, para ser aplicadas de

"manera exclusiva - con carácter general o particular - en los te-

"rritorios de las Indias Occidentales"."

De lo que se desprende que en la elaboración de los preceptos juridicos que formaron el nuevo derecho, intervienen dos clases de organismos, de un lado los de la Metrópoli, como son la Corona, el Real y Supremo Consejo de Indias y la Casa de Contratación de Sevilla, y de otro los organismos y autoridades de las Colonias, con facultad para ello, como los Virreyes. Audiencias, Cabildos. Consulados, Intendencias, etc.

Sin embargo que el nuevo derecho se elabora en España y en Indias Occidentales, la potestad o facultad legislativa correspondia a la Corona, y cuando se ejercita en las Colonias es por delegación y a nombre del Rey. necesitando siempre las normas por ellos dictadas la confirmación real.

En la formación de ese conjunto de normas juridicas intervienen factores históricos que estudiados nos explican la presencia de elementos espirituales, morales, materiales - económicos - que en alguna forma son determinantes de la orientación y finalidad de las reglas reguladoras de las nuevas situaciones creadas.

En efecto, el contenido y objetivo se relacicnan directamente con el fenómeno histórico del Descubrimiento, Conquista y Colonización de América, y del análisis de ese momento inicial, obtendremos las consecuencias y esquema de la legislación. Aceptado hoy, que la penetración en sus origenes, no fue empresa en la que tomara parte el Estado Español, ya que no se realizó con la intervención de elementos militares regulares y costeada por la Hacienda Real, sino más bien. fué empresa privada, con inclinación popular y con esfuerzo particular, hallamos el origen de alguno de los aspectos de la nueva legislación.

3 Ots Capdequi, José Maria: Manual de Historia del Derecho Español en las Indias $y$ dei Derecho propiamente Indiano. Edit. Losada, S. A. Buenos Aires, 1945. p. 25. 
Igualmente, admitido por los tratadistas que el Descubrimiento de América plantea una serie de problemas jurídicos, tanto en lo que se refiere a los justos títulos con que España defiende sus nuevos dominios y se empeña en justificar su penetración, como en cuanto a la situación de los aborígenes, hemos de aceptar que las normas que tratan de resolver aquellos problemas están condicionadas por el factor histórico y por elementos espirituales y materiales.

Y desde el momento que España invoca como justo título en la penetración las Bulas de Alejandro VI y evangelización de las nuevas tierras. amparando asi la conquista y colonización por la propagación de la fe, se imprime a la legislación un carácter ético y religioso, que permanecerá como una de sus notas esenciales.

A su vez desde el momento que España escucha la campaña de los frailes indianistas en favor de los naturales, que defienden los más elementales derechos de aquellos, crea un conjunto de normas protectoras, con un verdadero contenido social, que también es permanente en toda la evolución del nuevo derecho.

$Y$, finalmente, desde que se plantea la situación juridica de los nuevos dominios en relación a otros Estados y surge la solución de Vitoria se está sentando la base fundamental del Derecho Internacional en el siglo XVI.

Todos los elementos antes mencionados originan consecuencias juridicas vinculadas a determinados instrumentos públicos, entre ellos las Bulas de Alejandro $V I$, que constituyen la partida de nacimiento del Derecho Indiano, ya que son el origen y la confirmación del mismo, desde el momento que la Corona no solo admitió sino invocó como titulo legal del dominio español en América, esos instrumentos. ${ }^{2}$

Junto a las Bulas hemos de mencionar otros documentos no menos importantes y señalados por algunos como los originarios del nuevo derecho; ellos son las Capitulaciones, otorgardo por lo tanto a la fuente de origen un carácter contractual. Sin embargo conviene tener presente que las capitulaciones, existian en el Derecho Real de España antes del Descubrimiento de América y se emplearon por los Reyes para pactar con los particulares el desempeño de determinadas empresas. Pero en las Capitulaciones otorgadas por los Reyes en el caso de América, entran en juego una serie nueva de circunstancias que determinan que éstas exhiban un carácter peculiar, pues cada una de ellas podía ser diferente de la otra en cuanto a determinadas condiciones - no en cuanto el fondo- del pacto mismo, fueron especie de fueros - mutatis mutandi - que imprimieron a la legislación un carácter de particularismo juridico y en cierta forma de pritilegio.

- Zavala. Silvio: Las Instituciones juridicas en la Conquista de América. Madrid. 1935. pàg. 1. 
La mayor importancia de las Capitulaciones, reside en el hecho de ser ellas las primeras piedras de la organización de la administración territorial de las Indias Occidentales."

Es necesario también dejar establecido que, en alguna forma, el nuevo derecho muestra notas esenciales y elementos pértenecientes a la. Edad Media - ya superados en España - precisamente en las Capitulaciones, y la prueba de ello se tiene en las concesiones o gracias, en las facultades, en los titulos con carácter vitalicio. o por dos vidas, en la facultad de repartir tierras y solares y proceder a erección de fortalezas, etc., que contenía cada capitulación.

Otro punto importante es el relativo a la expansión del derecho castellano en Indias Occidentales, si este se opera o no a través del Derecho Indiano, tal vez, fue un propósito, pero la realidad histórica, geográfica y social determinó la elaboración de un cuerpo nuevo con normas propias y dictadas para casos concretos. con la tendencia a generalizarlas, y en las que se tomaron en muchos casos las instituciones y costumbres de los naturales. como base de la legislación, como lo demuestran los estudios, realizados por los juristas coloniales, de las instituciones indigenas.

Las normas del Derecho Indiano, dictadas para casos concretos como ya se señaló, no impidió la generalización, pero abundan las normas de derecho público, no asi las de derecho privado, lo que explica el porqué de la aplicación del derecho castellano, apesar de su carácter supletorio.

Tampoco puede negarse alguna tendencia de asimilar y uniformar la legislación indiana dentro de las distintas secciones de la América y aún con la castellana, pero la realidad histórica y social impuso otra cosa, con consecuencias juridicas diferentes, ya que en juego factores diversos imprimen variedades a cada región dentro del panorama general. Más, si se tiene en cuenta que las normas se dictaron también por los organismos y autoridades de la Colonia para resolver problemas de cada zona, que desde luego eran diferentes de los de otras, esta variedad de normas, que dá matiz propio a cada Derecho Nacional en América, es tema de apasionante investigación, pudiendo tenerse ya un ejemplo en la Encomienda, tan propia en el Perú y con caracteristicas distintas en Nueva España.

No está demás insistir en el sentido ético y religioso del nuevo derecho, explicado en parte por la polémica en torno al justo título, por la invocación de las Bulas de Alejandro VI por parte de los Monarcas españoles como fundamentación, legal de la penetración y por la interpretación de las mismas por los tratadistas de los dos ciclos, de todo lo cual se desprende la obligación de la Corona de evangelizar las tierras descubiertas, deber que se mantiene y finalidad que se sostiene en todo momento, en la legislación que se dicta para Indias Occidentales, como un firme propósito, en los tres periodos o momentos

Garcia Gallo. Alfonso: Los origenes de la Administracion, Territorial de las Indias. Madrid. 1944. 
de evolución, como lo hace notar don Niceto Alcalá Zamora. Lo que también demuestra el porqué de la intervención del teólogo y del moralista en la elaboración de los preceptos dictados, de manera principal con los Reyes Católicos.

Para concluir con las características del nuevo derecho, solo nos queda indicar que, apesar de ser un nuevo cuerpo de disposiciones, ellas respetaron y permitieron la supervivencia del derecho indigena, del cual recogieron algunas de sus instituciones, y procuran amoldar a la nueva situación las anteriores formas de su derecho.

Pero. frente a la exposición de los elementos fundamentales y de la orientación primordial y del contenido, es merester referirse a otro aspecto no menos interesante, éste es, la situación legal de los naturales, frente al nuevo estado de cosas.

No vamos a estudiar en este punto la inaplicación de las normas, sino el aspecto de las normas dictadas a su favor, y para ello hemos de admitir que la condición legal de los naturales, en alguna forma se encuentra condicionada a la polémica en torno a justo titulo y a soluciones propuestas para el mismo, en el que Silvio Zavala encuentra dos ciclos: el primero, basado en la exaltación de las jurisdicciones locales occidentales, - autoridad temporal del Papa y jurisdicción universal del Emperador - o en el ataque a la categoria de los naturales, por considerárseles como bárbaros, pecadores, infieles, viciosos, de lo que desprendian la necesidad que los naturales se sometieran. $\mathrm{Y}$ el segundo ciclo, en el que los autores, no aceptaron extender las jurisdicciones europeas y afirmaron y defendieron la condición humana de los indios y el respeto de sus derechos, no obstante ser gentiles.

Casualmente, a través de la discusión, surjen los defensores de los naturales, en la propia América, los frailes indianistas, entre los cuales destaca Fray Bartolomé de las Casas - tan discutido - con cuyos argumentos se consigue una verdadera influencia sobre los legisladores que se manifiesta en una serie de normas juridicas protectoras de los naturales, imprimiendo de esta manera al nuevo derecho un verdadero sentido social, que complementa así la preocupación de la Corona de defender y proteger a sus nuevos súbditos y a la vez que evangelizarlos.

No sería completo este cuadro si no se considerara otra de las consecuencias juridicas, directamente relacionada con la aplicación de los nuevos pre. ceptos que forman el Derecho Indiano. y ésta es, la inaplicación de la legislación en las Indias Occidentales, o en otras palabras, el profundo contraste entre la legislación escrita, cuya finalidad de propósito y contenido es manifiesta y la aplicación de la misma, - desde luego ineficaz.

La razón de este divorcio se debe a una serie de factores y elementos en iuego, admirablemente señalados por Toribic Esquivel y Obregón en sus "Apuntes para la Historia en México", cuando nos habla del español destructor de su derecho y del indio destructor de su ley, indicando como, al aceptarse la alta finalidad espiritual de la colonización. y no simplemente utilitaria - de- 
mostrada ya, por la defensa que algunos españoles, hacen de los naturalesy como al choque de dos derechos, el castellano y el indigena, se deforman, y de esta situación saltan las causas que explican la inaplicación de la ley, como son entre otras el afán de riquezas de los conquistadores y colonizadores. la lejania de la autoridad reguladora. y la mansedumbre de los naturales de un lado, y del otro por parte de los indios, como estos destruyen sus propias normas por aprovechar de las nuevas formas del derecho, que les permite escapar a sus antiguos preceptos, pero sin llegar a la adaptación de las nuevás.

Nada pudo el afán y el celo de la Corona, y de sus organismos Metropolitanos, y por qué no decirlo en muchos casos, el de las autoridades coloniales. lo mismo que la constante elaboración en el Supremo Consejo de Indias -órgano legislador - y el sentido de minuciosa reglamentación frente a los vicios antes mencionados que llegaron casi a anular el formidable esfuerzo que representa como contenido espiritual y social las normas del Derecho Indiano.

Queda por esbozar la consecuencia del Descubrimiento de la América en cuanto se refiere al Derecho Internacional.

Sabido es que, hasta hace algún tiempo; se aceptaba que el Padre del Derecho Internacional fue Grocio, pero felizmente esta tendencia se ha superado hoy, y se admite ya, que el verdadero creador de las bases de aquel derecho es el español Vitoria en sus "Relecciones" en 1526, cuando afirmaba que: "los "hombres son de naturaleza eminentemente limitada; un hombre no puede ha" cerlo todo. Los hombres se necesitan unos a los otros para poder empren" der labores que le impone su naturaleza intelectual y racional. Y de la mis" ma manera que los hombres no se bastan cada uno de ellos asi mismo las "naciones, cada una necesita de las otras", de lo que saca la siguiente conclusión:

"Las naciones no son independientes, sino interdependientes, es decir, unas " dependen de las otras. La cuestión fundamental por lo tanto, para las rela" ciones entre todas ellas, debe ser forzosamente la libertad de comercio"."

Vitoria, pertenece al segundo ciclo en la discusión sobre el justo título y no acepta la exaltación de los valores occidentales, esto es el podier temporal del Papado y la jurisdicción universal del Emperador, y busca y encuentra la justificación de la penetración española en otras razones a través de las cuales llega a la conclusión expuesta - no siendo desde luego este artículo. propio al desarrollo de la tesis de Vitoria - sentando las bases fundamentales del Derecho Internacional.

En conclusión no podemos negar un contenido espiritual y social al Derecho Indiano y a la vez reconocer que su origen, evolución y orientación está condicionada por los factores históricos del momento.

4 Toribio Esquivel Obregón: Hernán Cortés y el Derecho Internacional en el Siglo XVI. Edit. Polis. México, D. F. 1939. 
Niceto Alcalá Zamora, señala tres épocas en la Historia Legislativa de Indias, que son: la que corresponde al reinado de los Reyes Católicos y :omienzos del confuso poder de Doña Juana, Don Carlos y Don Felipe; la segunda: de la dinastía Austriaca, y la tercera: de la Borbónica; periodos a los que él mismo denomina: el Prólogo, el Texto y el Epilogo.

El primer periodo se encuentra vinculado estrechamente al descubrimiento y conquista, por lo tanto intimamente relacionado con los documentos juridicos que dan origen al nuevo derecho, tales como las Bulas Papales, las Capitulaciones, los Requerimientos, las primeras Instrucciones. las primeras Leyes Generales, etc.; orientando sus precəptos con un profundo sentido ético y religioso que garantice la libertad de los naturales, y dando a la legislación las notas esenciales que habian de mantenerse en los periodos posteriores, ya que reconocida la finalidad de la evangelización como fin, y el amparo de los derechos de los indios, se dictan las primeras normas, que son también el resultado de dos tendencias, la utilitaria, de los descubridores y conquistadores, y la espiritual de la Corona, que se empeña en que se de cumplimiento a sus disposiciones a favor de los indios, aunque con resultados contrarios.

Ese sentido espiritual de las primeras normas, nos dan la explicación de la presencia del sacerdote junto al jurista en la elaboración de la legislación.

También en este primer momento, como podemos notar, la aplicación de la legislación se realiza a medida que adelanta la conquista en los distintos territorios en momentos diversos, naciendo asi con un carácter territorial $y$ local.

La segunda etapa del desarrolio del Derecho Indiano corresponde, conforme a la clasificación de Alcalá Zamora, a la dinastía Austriaca, y a ella se concede el título de ser la que verdaderamente crea el nuevo derecho, tanto por el esfuerzo legislativo, cuanto por el impulso y orientación de la misma. Nota esencial de este periodo es, sin lugar a dudas, el haber mantenido en la organización territorial. los dos grandes núcleos de cultura indigena, sirviendo de base a la formación de los Virreynatos de Nueva España y el Perú.

A esta época pertenece también todo el proceso de integración del Derecho Indiano, manifestado a través de las tentativas, primero. y de las recopilaciones locales después, que culminarian con la preparación de las Leyes de Indias, hasta la formación de la Recopilación de 1680.

Asi también se acentúa otra de las notas caracteristicas, en el empeño de uniformar la legislación de Indias hasta llegar a la Recopilación; igual que la "tendencia centralizadora de la Corona"."

Casualmente en el proceso de integración del nuevo derecho, hemos da mencionar, entre otras, las recopilaciones locales, tales como el Cedulario de

- Basadre. Jorge: Historia del Derecho Peruano. Edit. Atenas. Lima. 1937. p. 241. 
Puga, para Nueva España; las Ordenanzas de Toledo, para el Perú; y, en España, el Código y el Cedulario de Encinas.

En el caso del Perú, aparecen, dentro del periodo en estudio, junto a lá Copilación general los esfuerzos locales, debidos a Escalona y Agüero con su "Gazophilacium" y Tomás Ballesteros con sus "Ordenanzas".

Fácil es pues darse cuenta de la importancia del periodo, llamado con toda propiedad del Texto, sin dejar de resaltar la obra de completar la organización territorial.

El tercer periodo, de los Borbones, aporta a la Legislación Indiana, no menos material y directivas que el anterior, y, algunas de sus pautas, verdaderas reformas de los sistemas encontrados.

En efecto, a este período debemos el haberse dejado a un lado la antigua demarcación de los dos tradicionales Virreynatos, y el haberse pasado a los cuatro, que han de ser encontrados al iniciäse las luchas por la Independencia, dejando asi huella para las demarcaciones republicanas.

Junto a la nueva demarcación territorial, se reforma su organización al establecerse las Intendencias que sustituyen a los corregimientos, dictándose para el efecto las Ordenanzas de Intendentes, en 1782, 1786 y 1803.

En lo que respecta al sistema de comercio, las reformas en contra del sistema imperante del monopolio son no menos notables, destacando entre ellas, las de 1774 que permitian el comercio entre el Perú. Nueva España, Nueva Granada y Guatemala; la de 1776 que extiende al comercio de Buenos Aires. los beneficios de la de 1774 y la promulgación del Reglamento de Aranceles Reales en 1778 para el comercio libre de España e Indias. Las mismas innovaciones se observan en la legislación de Minas y Aguas y Municipal.

Caracteristica saltante, como hace notar el tantas veces mencionado Niceto Alcalá Zamora, es la afirmación de poder personal. El poder de los virreyes, se verá aumentado desde 1752 al incorporarse al Virrey la Superintendencia en materias de Hacienda.

En en conclusión el periodo comentado, tan rico en notas, como notable en las huellas que deja en la evolución juridica de los pueblos americanos.

\section{III}

El caso del Perú en la evolución del Derecho Indiano encuadra, desde un punto de vista general, dentro de las propias formas de la evolución Continental, sin embargo, como ya se indicó, se puede buscar las notas de distinción que son saltantes para hallar el Derecho Indiaro Peruano.

Asi, por ejemplo, el nacimiento del Derecho Indiano Peruano se encuentra relacionado con el célebre contrato de Panamá, aunque su origen en realidad se hallaría con la Capitulación, otorgada por la Corona a Pizarro para la conquista del Perú, y también encontraremos un Requerimiento, el aplicado 
en Cajamarca, lo mismo que el reparto de solares y tierras y más tarde por expresa autorización el de encomiendas.

Por tanto podriamos encontrar cada una de las caracteristicas del Derecho Indiano Continental, pero, en nuestro caso, hay elementos o propios o más definidos que en otras secciones de América.

Asi, por ejemplo, en lo que respecta a la influencia o mejor hablando a la supervivencia de las instituciones indigenas en la legislación indiana, en el Perú, es mayor, y se encuentra marcada, por el interés de la Corona en la investigación sobre las normas de los pueblos conquistados, del que es caso típico. los trabajos de Polo de Ondegardo y Matienzo, y los trabajos durante Toledo.

De otro lado, las guerras civiles entre los conquistadores y los levantamientos de los encomenderos, determinaron modificaciones en la organización territorial peruana y en la legislación indiana, como se puede apreciar en la promulgación de las Nuevas Leyes de 1542, los mismos que en su posterior derogación, que en gran parte se debió a los sucesos del Perú.

En el proceso de Recopilación de las Leyes de Indias, el esfuerzo, tanto local, como general que se desprende del Virreynato Peruano, es muy notable y digno de ser considerado independientemente.

La apreciación de todos estos faciores, nos permitiria intentar, presentar una división de los Periodos del Derecho Indiano Peruano, marcada por los propios actos y hechos de nuestra evolución histórica y jurídica.

Esta división en etapas seria la siguiente:

Primer periodo, que llamaríamos Primitivo. El Derecho Indiano Peruano. comienza con la Capitulación de Toledo (1529) por la cual Pizarro obtiene la conquista del Perú. contando su jurisdicción una extensión de doscientas leguas de tierra a contarse por la costa, desde el pueblo de Zarumilla, hasta el pueblo de Chincha. Y por la misma se le concedieron entre otras mercedes, los títulos de Gobernador. Capitán General y Adelantado del Perú, con dependencia directa del Monarca - sin referirnos a las mercedes a Almagro, Luque $y$ otros conquistadores.

Los documentos y actos jurídicos más importantes de este periodo son, sin lugar a dudas, La Capitulación, el Requerimiento - de Cajamarca-, las Actas de fundación de las primeras ciudades, entre las que están la toma de posesión y fundación de la ciudad del Cuzco, la fundación de Trujillo, Lima, etc., al reparto: de botin. de solares, tierras e indios, las disposiciones ampliatorias de la Capitulación conteniendo las primeras normas de organización y ampliando las facultades de Pizarro, entre las que contamos la Cédula que autoriza a Pizarro para entregar encomiendas, - sin embargo que, de hecho, las habia repartido con anterioridad - la introducción de los negros y el establecimiento de los Cabildos. 
Sus notas esenciales estarian representadas, por un origen contractual, en el Derecho Indiano Peruano - el contrato de Panamá, la Capitulación de Toledo- por la aplicación de la tesis de la exaltación de los valores europeos -el Requerimiento de Cajamarca - por la presencia de un verdadero Derecho Premial, con el reparto de botin, tierras, indios, y con las mercedes y gracias otorgadas por la Corona; en ciertaa forma su tendencia $f$ cudal. - con la forma y fondo de la Capitulación - su origen territorial y local como ocurrió en otras secciones de América, consecuencia del progreso escalonado de la conquista y población.

En este primer periodo encontraremos una forma aplicada al Perú para justificar la penetración, y ella es la exposición que se hace sobre la tirania de Atahualpa.

La organización territorial primitiva es imprecisa, y sus consecuencias, son las guerras civiles entre los conquistadores. 10 mismo que las facultades, que se van ampliando progresiva y paulatinamente. La forma de administración se concentra en el Gobernador.

Las consecuencias juridicas más importantes: las guerras civiles, el reparto de encomiendas, el nacimiento de fuertes intereses económicos en torno a ellas, la inaplicación de la legislación indiana, con el desconocimiento de los derechos de los indios.

Es primer periodo se prolonga hasta las Nuevas Leyes de 1542.

Segundo periodo, de 1542 a 1596, que llamaremos de Organización. se inicia con la promulgación de las Nuevas Leyes de 1542 y con la designación de Virrey y miembros de la Audiencia del Perú.

En efecto, la promulgación de las Nuevas Leyes, modifican el sistema de organización politico, administrativo y territorial del Perú, desde el momento que se establece el Virreynato y la Audiencia en Lima, y luego se nombra Virrey para el Perú: y además el régimen de las encomiendas queda alterado. lo que determinaria efectos trascendentales en este Virreynato."

En este momento se desarrollan acontecimientos históricos que influyen poderosamente en la evolución de! Derecho Indiano Continental. tales como el levantamiento de Gonzalo Pizarro, que representa los intereses de los encomenderos, lo que obliga a la Corona a la derogación de aquellas disposiciones de las Nuevas Leyes, que afectaban directamente a las Encomiendas.

Sin embargo, posteriormente, sofocado ya, el otro levantamiento. de Francisco de Hernández Girón. que fuera causado por la insistencia de la Corona en suprimir el servicio personal de los indios, los dominios de España, ingre

* Véase las Nuevas Leyes 1542-43.-Reproducción de los ejemplares existentes en la Sección Patronato del Archivo General de Indias. transcripción y notas por Antonio Muro Orejón-Consejo Superior de Invest. Cientificas. Esc. de Est. Americanos de la Univ. Sevilla. 1945. 
san en una etapa de verdadera organización y reglamentación, de la que son notables representantes en et orden civil en el Perú, el Virrey Toledo y en al orden eclesiástico el Santo Arzobispo Toribio de Mogrovejo.

En cuanto al aspecto puramente legislativo, fuera del valor de las Nuevas Leyes, señaladas por Jorge Basadre, como la primera tentativa de coordinación de las Leyes de Indias,' y ampliadas por las Ordenanzas de 1543 para el buen tratamiento de los naturales, hemos de mencionar las Ordenanzas de Nuevos Descubrimientos y Poblaciones, promulgadas por Felipe II en 1573.

Igualmente corresponden a este momento los primeros pasos a favor de ia recopilación, que tienen un sentido local, tanto en Nueva España como en el Perú, y cuyas manifestaciones son, el Cedulario de Puga, y entre nosotros las famosas "Ordenanzas" de Toledo, en las que trabajan los juristas Polo de Ondegardo y Matienzo, que, con su sentido de reglamentación, legislan sobre distintas materias, especialmente: reducciones de indios, corregidores, jueces, repartimientos, adquisición de propiedad por los españoles, facultades de los caciques. Hacienda Real, yanaconazgo, mitas, trabajo en minas, etc.

No menos amplia fue la labor del Consejo de Indias al dictar numerosas normas sobre organización de los dominios de España en la América.

El periodo de reglamentación se refleja en todas las instituciones de ta que es un caso la Encomienda, en la que después del reparto de Guaynarima y problema de la Perpetuidad se encausa por una serie de preceptos de organización."

El juego de todos estos elementos permitieron superar el primitivo sentido local y territorial por el de la generalización y una tendencia centralizadora en la legislación indiana, coronada por la profunda reglamentación y la iniciación del proceso de recopilación territorial.

Termina esta etapa con los esfuerzos generales de recopilación con el Código Ovandino (1571). los trabajos de Alonso de Zorita (1574), y la publicación del Cedulario de Encinas (1596).

Tercer Periodo, de 1596 a 1680 , de Preparación de la Recopilación.

En el se fijan definitivamente las bases de organización de Indias Occidentales, y el Virreynato del Perú alcanza su esplendor territorial, ya que comprende casi todas las colonias españolas de América del Sur, dependiendo de este Virreynato, los Gobiernos de Chile y Buenos Aires, la Presidencia de Quito, la Región Amazónica, lo que significa ser el dominio más vasto del mundo.

Esta época es la segunda de preparación de la recopilación de las Leyes de Indias, con la intervención de juristas como. Diego de. Zorrilla, Rodrigo de Aquiar y Acuña, y los relacionados estrechamente con el Perú: Solórzano y

Basadre Jorge: Historia del Detecho Peruano. Edit. Antena. S. A. Lima. 1937. p. 241. Belaunde. Manuel: La Encomienda en el Perú. Lima, 1945. 
Pereira y León Pinelo; es sin lugar a dudas, la etapa de verdadera integración del Derecho Indiano, hasta su culminación con la Recopilación de Leyes de Indias de 1680 .

No está ausente la nota esencialmente peruana, ella es un grano más en el proceso de recopilación local, con la obra de Escalona y Agüero: "Gazophilacium Regiun Peruvicum" (1647) que trata sobre materias de Hacienda.

Aparecen también, las mejores obras de doctrina del Derecho Indiano, como "Politica Indiana", escrita y publicada primero en latín: "De Indiarum Jure" (Tomo I, 1629; y Tomo II, 1639) la que luego fue ampliada y traducida por su autor (Madrid, 1649), la más extensa y completa sobre legislación indiana; junto a ella la de Antonio León Pinelo: "Trata de las Confirmaciones Reales de Encomiendas" (1630).

El aporte peruanista está presente con Pareceres y Memoriales que se refieren o al problema de las Encomiendas o a la situación legal del indio. Asi" tenemos el del franciscano Fr. Miguel de Agia, "Tratado que contiene tres pareceres graves de Derecho" (Lima, 1604) y el "Memorial que trata de la Perpetuidad de las Encomiendas de Indios en el Perü" (Madrid, 1617), del que es autor don Juan Ortiz de Cervantes, Abogado de la Audiencia de Lima, y Procurador de la Ciudad del Cuzco; y junto a ellos el del Alcalde del Crimen de Lima: "Trabajos, agravios e injusticias que padecen los indios del Perú, en lo temporal y espiritual" (1657).

En resumen, puede afirmarse que esta etapa es la del verdadero proceso formativo del derecho indiano que culmina con el mayor monumento legislativo de entonces: La Recopilación de 1680, vigente desde 1681.

Cuarto periodo, de 1680 a 1792, de aplicación de la Recopilación de 1680. y esfuerzos por el Nuevo Código; en ella no podía faltar el elemento peruano. con las "Ordenanzas de Ballesteros" (1685), que reunen las órdenes, ejecutorias, provisiones, y cédulas reales posteriores a las Ordenanzas de Toledo, conteniendo por tanto el régimen legal de la Colonia hasta mediados del siglo XVII.

Este período queda en gran parte encerrado dentro del Gobierno de los Borbones; por lo tanto sus caracteristicas, ya han sido fijadas al estudiar este aspecto y al dejar establecido que la huella de esta dinastia es notable, ya en lo que se refiere a la organización territorial con desdoblamiento de los virreynatos, como con las reformas en el sistema politico y económico lo mismo que en lo que respecta al derecho de Minas, Agua, aplicación de las Ordenanzas Militares (1728 y 1788 ), Navales (1793 y 1802$)$, etc.

A la vez se efectuan los nuevos trabajos y esfuerzos por dotar a las colonias de un nuevo Código, para lo que se forman las comisiones respectivas, llegando al proyecto de Ansotegui y posteriormente al que la Junta formó del Libro I del Nuevo Código, aprobado en marzo de 1792, aunque en realidad no llegó a aplicarse. 
Le este momento es la aparición de la obra del panameño Manuel José de Ayala, oficial de la Secretaría del Despacho de Indias, quien escribió "Diccionario de Gobierno y Legislación de Indias" cuyo "Indice" se publicó en Madrid en 1778 .

La peruanidad del periodo, la tenemos en los "Reglamentos" de los que son muestra el formado por el Deán Saavedra, en 1700, para el valle de Trujillo y "El tratado general sobre las aguas que fertilizan los valles de Lima" del Oidor Ambrosio Cerdán de Landa. No está demás recordar que en el Perú se reformó el sistema de organización politica, con el establecimiento de las Intendencias en 1784.

El quinto y último periodo, de 1792 a 1821 , tan relacionado ya con la agitación revolucionaria, en la que si apenas destacaran como instrumentos politico importante la Constitución de 1812, que modifica la condición política de Indias. $Y$, en cuanto al esfuerzo privado de recopilación, el Catálogo de Juan de Matraya y Ricci (Lima, 1819). Con lo que se puede decir que se cierra el periodo y el Derecho Indiano Peruano en 1821, con la Declaración de la Independencia.

\section{LIBRERIA "STUDIUM"}

AMARGURA 954 - Apartado 2139 - Teléfono 35993 - L I M A

Tiene constantemente en venta las mejores obras de

Derecho - Literatura - Historia - Filosofía 